\title{
Experimental Infection of Asian House Geckos (Hemidactylus Frenatus) With Enterococcus Lacertideformus Demonstrates Multiple Routes of Disease Transmission and The Relative in-Vivo Efficacy of Antibiotic Treatments
}

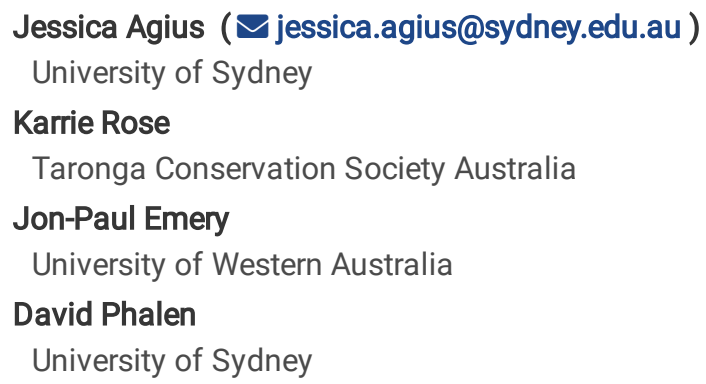

\section{Research Article}

Keywords: Antibiotics, Asian house gecko, Bacteria, Biofilm, Enterococcus, Experimental infection, Reptiles, Treatment

Posted Date: January 20th, 2021

DOI: https://doi.org/10.21203/rs.3.rs-147441/v1

License: (c) (1) This work is licensed under a Creative Commons Attribution 4.0 International License. Read Full License

Version of Record: A version of this preprint was published at Scientific Reports on July 5th, 2021. See the published version at https://doi.org/10.1038/s41598-021-92999-y. 


\section{Abstract}

The disease caused by Enterococcus lacertideformus is multisystemic and ultimately fatal. Since its emergence, the disease has significantly impacted the captive breeding programs of the Extinct in the Wild Christmas Island Lister's gecko (Lepidodacty/us listeri) and blue-tailed skink (Cryptoblepharus egeriae). The bacterium's pathogenicity, inability to grow in-vitro, and occurrence beyond the confines of Christmas Island necessitated the development of an experimental infection and treatment model. Asian house geckos (Hemidactylus frenatus) were challenged with a single dose of $E$. lacertideformus either by mouth, application to mucosal abrasion or skin laceration, subcutaneous injection, coelomic injection, or via co-housing with an infected gecko. Five healthy geckos acted as controls. Each transmission route resulted in disease in at least $40 \%(n=2)$ geckos, expanding to $100 \%(n=5$ groups) when E. lacertideformus was applied to skin laceration and mucosal abrasion groups. Incubation periods post-infection ranged between 54 and 102 days. To determine the efficacy of antibiotic treatment, infected geckos were divided into six groups (enrofloxacin 10mg/kg, PO, q24, amoxicillin clavulanic acid 10 $\mathrm{mg} / \mathrm{kg}, \mathrm{PO}, \mathrm{q} 24$, enrofloxacin $10 \mathrm{mg} / \mathrm{kg}$ combined with amoxicillin clavulanic acid $10 \mathrm{mg} / \mathrm{kg}, \mathrm{PO}, \mathrm{q} 24$, rifampicin 15mg/kg, PO, q24, clarithromycin $15 \mathrm{mg} / \mathrm{kg}, \mathrm{PO}, \mathrm{q} 24$, and untreated controls) for 21 days. Response to treatment was assessed by change in lesion size, bacterial dissemination, and histological evidence of a host immune response. Irrespective of the antibiotic given, histology revealed that geckos inoculated by skin laceration were observed to have more extensive disease spread compared to other inoculation routes. The reduction in the average surface area of gross lesions was $83.6 \%$ for geckos treated with enrofloxacin, followed by the combination therapy amoxicillin clavulanic acid and enrofloxacin (62.4\%), amoxicillin clavulanic acid (58.2\%), rifampicin (45.5\%), and clarithromycin (26.5\%). Lesions in geckos untreated with antibiotics increased in size between $100 \%$ to $300 \%$. In summary, enrofloxacin and amoxicillin clavulanic acid show promising properties for the treatment of $E$. lacertideformus infection in geckos. The Asian house gecko $E$. lacertideformus infection model therefore provides foundational findings critical to the development of effective therapeutic and prophylactic treatment protocols aimed at conserving the health of infected and at-risk reptiles.

\section{Introduction}

Lister's geckos (Lepidodactylus listeri) and blue-tailed skinks (Cryptoblepharus egeriae), once abundant on Christmas Island, are now Extinct in the Wild ${ }^{[1]}$. These Critically Endangered lizards are maintained only in conservation breeding facilities on Christmas Island and at Taronga Zoo, Sydney, Australia. The breeding programs for both species on Christmas Island are threatened by a recently emerged bacterium, Enterococcus lacertideformus, which has caused two outbreaks in the Christmas Island captive breeding facility. The initial outbreak of $E$. lacertideformus led to deaths of more than 40 Lister's geckos and ten blue-tailed skinks in the breeding enclosures. The subsequent outbreak of the disease occurred in partially enclosed outdoor exclosures housing the blue-tailed skink male breeding stock, resulting in the deaths of more than 30 individuals. Both outbreaks of the disease were likely initiated by direct contact with infected free-ranging invasive reptiles and the outbreaks were ultimately controlled by depopulation of affected and in contact lizards. Treatment was not considered an option at the time because the susceptibility of E. lacertideformus to antibiotics was not known [2].

Untreated disease caused by E. lacertideformus is uniformly fatal. Animals infected with E. lacertideformus initially exhibit swellings composed of a subcutaneous white gelatinous material predominately localised to the face that subsequently disseminate, forming often coalescing nodules in multiple organ systems. Microscopically the lesions are composed of bacteria that are suspended in a thick biofilm. Bacterial aggregates grow by expansion, replacing the surrounding normal tissue and causing bone lysis. In most instances the lesions are not accompanied by inflammation, although uncommonly, aggregates of lymphocytes are found in the tissues adjacent to the lesions. The course of the disease is slow but progressive, with infected lizards surviving three weeks to four months after the initial lesions are observed

[2]. How E. lacertideformus is acquired is not known. However, given that the initial lesions develop on the face, it is possible that infection occurs through bite wounds from other lizards or following colonisation of the oral cavity from an environmental source. To date, $E$. lacertideformus has not been grown in-vitro using traditional bacterial isolation techniques ${ }^{\text {[2] }}$

Enterococcus lacertideformus is enzootic in wild reptiles on Christmas Island. Surveys of the free-ranging invasive mute (Gehyra mutilata) and Asian house geckos (Hemidactylus frenatus) found animals infected with E. lacertideformus at multiple sites across the island over a period of four years ${ }^{[3]}$. Thus, the organism poses a continued threat to both the captive breeding program for the Lister's gecko and bluetailed skinks on Christmas Island and any effort to release these species back into the wild.

It is also likely that E. lacertideformus is not confined to Christmas Island and threatens other species of reptiles. A morphologically identical bacterium causing indistinguishable gross and microscopic was described in Singapore house geckos (Gekko monarchus) in Asia ${ }^{[4]}$, and in five species of lizards from Europe [5]. Both studies ascribed the agent to the Streptococcus genus of bacteria, however, at the time of publication, enterococci were classified within that genus. Efforts to culture the organisms were unsuccessful [4,5]. More recently, free-ranging brown anoles (Anolis sagrei) in Florida, United States of America were also observed with morphologically identical facial and multisystemic 
microscopic lesions. Amplification of a 1,400 bp segment of the $16 \mathrm{~s}$ rDNA gene from DNA extracted from these lesions revealed that it was $100 \%$ identical to E. lacertideformus ${ }^{[6]}$.

Given the uniformly fatal nature of infection and the vulnerable nature of insular reptile species, disease mitigation strategies are required. Therefore, understanding the modes of transmission, clinical course of infection, and susceptibility of $E$. lacertideformus to antimicrobial treatment are critically important to inform disease control and management. Based on a recent metagenomics study ${ }^{[7]}$, it appears that $E$. lacertideformus has limited antibiotic resistance and is likely to be susceptible to fluoroquinolones, macrolides, broad-spectrum penicillins and rifamycins, antimicrobials that are effective against other enterococci and have the added advantage of high penetrating capacity of biofilms ${ }^{[8,9]}$. These antibiotics have also been shown to be safe when administered to reptiles and can be given orally, which is a significant advantage when treating reptiles as small as two grams. Additionally, single-administration pharmacokinetic trials using orally administered enrofloxacin in the Asian house gecko show that with appropriate dosages, therapeutic plasma concentrations can be achieved.

The aims of this study are twofold. The first is to identify a reproducible experimental model of infection that can shed light on the epizootiology and disease dynamics of this bacterium. The second aim is to assess the efficacy and practicality of five antibiotic treatment protocols that could be used to treat individual reptiles or captive populations of reptiles infected with $E$. lacertideformus.

\section{Materials And Methods}

\section{Animal ethics}

The research protocol and use of wild reptiles were approved by the University of Sydney Animal Ethics Committee (AEC) (2018/1380) on 16 July 2018 in compliance with the NSW Animal Research Act 1985, and the Australian code for the care and use of animals for scientific purposes. The authors complied with the ARRIVE guidelines 2.0 for the reporting of animal research conducted in this study ${ }^{[10]}$.

\section{Acquisition of experimental geckos}

\section{Disease-free geckos}

Asian house geckos were collected from a location on Christmas Island (10²8'20.4"S 105³4'44.2"E) where infected geckos have never been observed. The geckos were physically inspected for lesions characteristic of E. lacertideformus infection and their body condition score was recorded. Body condition, range 1 (poor) to 5 (well-muscled) was determined by the amount of muscle coverage surrounding the tail and dorsal pelvis. Only geckos that appeared healthy and had a body condition score of three or greater were used in the study. Geckos that did not meet these criteria were euthanised. Thirty-five mature (18 males, 17 females) geckos were randomly assigned to seven treatment groups of five animals using the random integer generator routine numpy.random.randint in Python v3.8 ${ }^{[11]}$, and acclimatised for 10 days. A sample size of five per treatment group was determined to be sufficient to detect a route of infection that resulted in a $50 \%$ infection rate using the formula: $n=\left[1-(1-P 1)^{1 / d}\right][N-((d-1) / 2)]$, where $n$ is the sample size, $P$ is the probability of detecting at least one case of the disease if it is present in the population [0.95], $\mathrm{N}$ is the population size [10,000], and $\mathrm{d}$ is the number of detectable cases in the population; where $\mathrm{d}=$ population size $x$ prevalence $x$ sensitivity of the test $[10,000 \times 0.5 \times 0.95]^{[12]}$.

\section{Naturally infected geckos}

For the infection trial, five naturally infected Asian house geckos demonstrating characteristic lesions of $E$. lacertideformus were captured by hand from a location on Christmas Island $\left(10^{\circ} 25^{\prime} 55.7^{\prime \prime} \mathrm{S} 15^{\circ} 40^{\prime} 13.4^{\prime \prime} \mathrm{E}\right)$ known to have a high prevalence of E. lacertideformus infection. Three of the five affected geckos were euthanised and their tissues harvested to prepare the inoculum, while the remaining two geckos (1 male, 1 female) were assigned to the infection by contact trial. An additional 10 Asian house geckos naturally infected with $E$. lacertideformus were captured at the same location and used in the antibiotic treatment trial.

\section{Experimental infection model}

\section{Housing and husbandry}

Geckos were individually housed in PenPal terrariums $(30.0 \mathrm{~cm} \times 18.0 \mathrm{~cm} \times 18.0 \mathrm{~cm})$ (Living World® PenPals). Only geckos allocated to the 'infection by contact' treatment were housed as pairs. Each terrarium contained a large $(18.0 \mathrm{~cm} \times 3.5 \mathrm{~cm})$ and small $(15.0 \mathrm{~cm} \times 2.0 \mathrm{~cm}) \mathrm{hide}$ made of PVC pipe, modified to sit stably on the floor of the enclosure. Both PVC pipes were sealed at one end with screen wire mesh to aid in capturing the gecko for twice weekly monitoring. Artificial foliage $(15.0 \mathrm{~cm} \times 10.0 \mathrm{~cm})$ was provided as an additional form of refuge. Water was supplied in a polypropylene lid and was refreshed daily. Geckos were misted with clean water twice weekly and fed three days per week 
with a mixture of live termites, moths, and crickets. All housing was contained within an escape-proof facility where the ambient temperature ranged from 26 to $30^{\circ} \mathrm{C}$.

\section{Infection trial}

Oral and skin swabs (Dacron, AMSL Scientific) were collected from all presumed disease-free and naturally diseased geckos prior to inoculation to confirm the absence or presence of E. lacertideformus infection via a real-time (qPCR) assay.

A fine needle aspirate (FNA) was collected from the lesions of five naturally infected geckos and subjected to cytology (Gram-positive cocci in chains encapsulated by a thick, lightly staining mucoid matrix), and qPCR to confirm E. lacertideformus infection. Diseased tissues from the head and oral mucosa of the infected lizards were aseptically collected and homogenised in phosphate buffered saline ( $\mathrm{pH} 7.5 \pm 0.5$; Sigma-Aldrich). The homogenised solution was serially diluted to achieve a concentration of approximately $4 \times 10^{6}$ organisms/mL. All experimentally infected geckos were inoculated with $0.02 \mathrm{~mL}$ ( 80,000 organisms).

The infection trial included six routes of inoculum delivery (Table 1). Geckos in the control, oral cavity, and subcutaneous groups were inoculated using manual restraint. For mucosal abrasion and skin laceration, and coelomic cavity challenges, geckos were immobilised with a subcutaneous injection of alfaxalone $(5 \mathrm{mg} / \mathrm{kg}$, Alfaxan, Jurox Animal Health) caudal to the left shoulder prior to inoculation. Following inoculation, all geckos were immediately returned to their enclosures. Sedated geckos were monitored for 20 minutes to ensure adequate recovery.

Table 1

The treatment groups used in the experimental infection trial.

\begin{tabular}{|c|c|c|c|c|}
\hline Treatment & Route & $\begin{array}{l}\text { Bacterial } \\
\text { Dose* }\end{array}$ & $\begin{array}{l}\text { Inoculum } \\
\text { Volume } \\
\text { (mL) }\end{array}$ & Site of Inoculation \\
\hline Control & SC & 0 & 0.02 & $\begin{array}{l}\text { Saline injection into the loose skin } 2 \mathrm{~mm} \text { off the ventral midline, and } 5 \mathrm{~mm} \text { cranial to } \\
\text { the pelvis. }\end{array}$ \\
\hline Oral cavity & PO & 80,000 & 0.02 & Direct administration into the oral cavity. \\
\hline Subcutaneous & SC & 80,000 & 0.02 & Subcutaneous injection into the loose skin at the medial aspect of the neck. \\
\hline $\begin{array}{l}\text { Mucosal } \\
\text { abrasion }\end{array}$ & & 80,000 & 0.02 & $\begin{array}{l}\text { Shallow abrasion of the gingiva on the right side of the face using a cotton swab. } \\
\text { Inoculum applied to abraded surface. }\end{array}$ \\
\hline $\begin{array}{l}\text { Skin } \\
\text { laceration }\end{array}$ & & 80,000 & 0.02 & $\begin{array}{l}\text { Shallow skin laceration ( } 1 \mathrm{~mm} \text { deep and } 5 \mathrm{~mm} \text { long) on the right lateral side of the } \\
\text { face rostral to the ear ostium. Inoculum applied to wound surface. Wounds covered } \\
\text { by a light-weight adhesive bandage for } 24 \text { hours. }\end{array}$ \\
\hline $\begin{array}{l}\text { Coelomic } \\
\text { cavity }\end{array}$ & IC & 80,000 & 0.02 & Co-housed with an infected animal for ten days. \\
\hline Co-housing & & $\mathrm{n} / \mathrm{a}$ & $\mathrm{n} / \mathrm{a}$ & Natural infection means. \\
\hline
\end{tabular}

All experimentally challenged geckos were held for a maximum of four months or until characteristic signs of E. lacertideformus developed, whichever occurred first. Oral swabs, and FNAs expressed onto swabs were collected from geckos that developed gross lesions to cytologically confirm E. lacertideformus infection. Infected geckos were enlisted into the antibiotic treatment trial. Four geckos that developed lesions were untreated, held for 28 days and euthanised, constituting an untreated control group.

\section{Infection by contact trial}

During the infection by contact phase of the infection trial, two affected geckos (a male and a female) were initially individually co-housed for 10 days with a disease-free gecko of the opposite sex. Following 10 days, the two affected geckos were then co-housed with a single disease-free gecko of the same sex for 10 days. The affected male was then exposed to a third gecko; a disease-free male for a period of 10 days. Following completion of this trial, both affected geckos were euthanised and diseased tissues collected to confirm that the lesions were caused by E. lacertideformus. During the co-housing phase, geckos were observed daily to monitor for bite wounds or other evidence of aggressive behaviour. All five geckos exposed to the infected geckos were held for four months.

\section{Monitoring}

All geckos used in the infection trial were observed daily in their enclosures. Twice weekly the lizards were weighed and visually inspected. Body condition score, food consumption, and faecal production were recorded, in addition to any observations that might relate to their 
health or disease status. When animals developed lesions characteristic of E. lacertideformus disease (e.g. facial swelling, epidermal nodules, and mass formation in the coelomic cavity or within viscera as determined by transillumination), the progression of their lesions were measured and photographed each time they were monitored.

\section{Endpoint and euthanasia}

During the infection and treatment trials geckos were euthanised prior to the end of the trial. Euthanasia of experimental geckos was undertaken if animals met the euthanasia end-point criteria, which included (1) a reduction of body condition score to two or less, or (2) facial or other swellings that interfered with normal activity, or (3) decreased appetite or anorexia. Geckos were euthanised with a subcutaneous injection of alfaxalone into the triceps musculature, resulting in an overdose of the anaesthetic agent. Once immobilised and unresponsive, lizards were decapitated.

\section{Experimental treatment trial}

\section{Therapeutic trial}

Geckos that developed E. lacertideformus disease (confirmed by cytology and qPCR) during the infection trial ( $\mathrm{n}=9$ ) and wild-caught geckos naturally infected with the disease $(n=10)$ were randomly allocated to each of the five antibiotic treatment groups, using the random integer generator routine numpy.random.randint in Python v3.8 ${ }^{[11]}$. The five antibiotic treatments consisted of: enrofloxacin, $10 \mathrm{mg} / \mathrm{kg}$, PO, q24; rifampicin, 15 mg/kg, PO, q24; clarithromycin, 15 mg/kg, PO, q24; amoxicillin clavulanic acid 10 mg/kg, PO, q24; and a combination of enrofloxacin, $10 \mathrm{mg} / \mathrm{kg}$ and amoxicillin clavulanic acid $10 \mathrm{mg} / \mathrm{kg}, \mathrm{PO}, \mathrm{q} 24$. Four geckos were allocated into each treatment group, with the exception of the combined therapy (enrofloxacin and amoxicillin clavulanic acid) group that only contained three geckos as a result of limited number of available animals. Treatment was administered to geckos seven days after the first signs were observed. Geckos were treated for a total of 21 days and then euthanised, although some animals were euthanised earlier if end-point criteria were reached. All animals in the treatment trial were monitored as described for the infection trial. Lesions were described, photographed, and measured twice weekly on their longitudinal and transverse axes. As a means of assessing antibiotic efficacy, the percentage change of the lesion was calculated for each gecko by comparing the surface area of the lesions at day 7 after signs were observed (day 1 of antibiotic treatment) and at euthanasia (day 21 of antibiotic treatment). For each gecko, the total surface area of the lesion(s) was determined before and after treatment. The change in lesion surface area was determined by $100 \% \times(A 2-A 1) / A 1$, where A2 was the surface area after treatment and $\mathrm{A} 1$ was the surface area before treatment. The percent change was then grouped for each antibiotic treatment and no treatment. $A$ boxplot was generated to compare the percent change in the lesion size across different treatments using the pandas library ${ }^{\mathrm{v} .1 .2 .0}{ }^{[13]}$ in Python v3.8 ${ }^{[11]}$.

\section{Sample collection and processing}

During post-mortem examination, oral swabs, a single liver lobe (as disease spread to the liver was commonly observed in naturally infected lizards), and a mid-sagittal section of the head were taken and stored in 100\% ethanol for qPCR analysis. Tissues with suspected $E$. lacertideformus lesions were also collected and stored in RNA-later (Sigma-Aldrich) and frozen for qPCR analysis. The remains were fixed in $10 \%$ neutral buffered formalin for histological examination.

\section{DNA extraction}

Prior to DNA extraction, oral swabs $(n=107)$, skin swabs $(n=45)$, lesion FNA swabs $(n=22)$, and tissues $(n=50)$ were rehydrated with four phosphate-buffered saline washes to remove residual fixative. Tissues were mechanically ground and digested with proteinase $\mathrm{K}$ for 3 hours. Pure genomic DNA was extracted from swabs using the buccal swab protocol from the QIAamp DNA mini extraction kit (Qiagen), and DNA was extracted from suspected E. lacertideformus diseased tissues using the animal tissue protocol from the DNeasy Blood and Tissue Kit (Qiagen).

\section{Quantitative PCR (qPCR) development and validation qPCR primers, probes, controls, and conditions}

The Primer-BLAST (NCBI) tool ${ }^{[14]}$ was used to design a primer and probe set specific to a short fragment of the E. lacertideformus glucose-6phosphate dehydrogenase (gdh) house-keeping gene (Table 2). Oligonucleotides were synthesised by Integrated DNA Technologies (IDT, USA). The fluorescein (5'6-FAM) labelled probe was assigned to hybridise with a complementary region between the forward and reverse primers. 
Table 2

Primer and probe set used in the Enterococcus lacertideformus-specific qPCR assay.

\begin{tabular}{|llllll|}
\hline Sequence ID* & $\mathbf{5}^{\prime} \diamond \mathbf{3}^{\prime}$ Sequence & Length $(\mathrm{bp})$ & $\operatorname{Tm}\left({ }^{\circ} \mathrm{C}\right)$ & $\mathbf{G C}(\%)$ & Amplicon Length (bp) \\
\hline EntL - Forward & CCAAATAATAGATGCGATTCCC & 22 & 59 & 40.9 & 171 \\
\hline EntL - Reverse & CTACTATCCAGTCACTCAATCC & 22 & 59 & 45.5 \\
\hline EntL - Probe & TGGGTTGAATCATTGACATCGTGAGA & 26 & 66 & 42 \\
\hline
\end{tabular}

Primers were optimised by testing forward and reverse primer concentration combinations of 150,300, 600, and $900 \mathrm{nM}$ with a fixed probe concentration of $250 \mathrm{nM}$. The primer:probe concentration that was most efficient, yielding the lowest quantification cycle (Cq), lowest variation in replicates, and negative no template control (NTC) was chosen. Primer annealing temperatures were optimised stepwise by increasing the annealing temperature from 54 to $64^{\circ} \mathrm{C}$ in increments of $2{ }^{\circ} \mathrm{C}$. The annealing temperature with the lowest $\mathrm{Cq}$, highest reproducibility between replicates, detection of the target DNA, and a negative NTC was chosen.

\section{qPCR specificity}

The specificity of the oligonucleotides was confirmed by scanning them against the National Center for Biotechnology Information GenBank database using the basic local alignment search tool ${ }^{[15,16]}$. A DNA panel of non-target bacterial species (including near relatives of $E$. lacertideformus) was used to validate the specificity of the qPCR assay. The non-target controls were Enterococcus villorum F1129D, Enterococcus faecium AUS0085, Enterococcus faecalis ATCC 29212, Staphylococcus aureus NCTC 6571, Escherichia coli NCTC 10418, and Pseudomonas aeruginosa ATCC 27853. Additional non-target isolates included vancomycin-resistant Enterococcus faecium, Enterococcus durans, Enterococcus hirae, Enterococcus villorum, Streptococcus anginosus, Streptococcus Group F, and Streptococcus Group G that were all isolated at the Taronga Zoo clinical pathology laboratory.

\section{qPCR efficiency, reproducibility, and limit of detection}

A double stranded $499 \mathrm{bp}$ artificial gBlock fragment was designed and synthesised by Integrated DNA Technologies (IDT, USA) to determine the limit of detection (LOD); the absolute minimum number of copies detectable by the assay. The gBlock fragment contained a partial sequence of the gdh gene (target template spanning 165 to $335 \mathrm{bp}$ of the gBlock fragment).

The LOD was determined using a 10 -fold dilution series of the gBlock from $1.22 \times 10^{8}$ to 1.22 gene copies $/ \mu \mathrm{L}$. Each dilution series was run in five replicates. The lowest concentration of gBlock that produced a $\mathrm{Cq}$ value in all five replicates was considered the LOD. Quantification cycle values over 38 were removed from the analysis and classed as non-detectable. Amplification efficiency was determined by plotting the Cq values versus the gBlock dilution and calculating the linear slope. The coefficient of determination $\left(R^{2}\right)$ was also calculated from this data.

\section{Enterococcus lacertideformus detection qPCR diagnostic assay}

DNA extracted from alcohol-fixed swabs, FNAs, and tissue samples were screened using the E. lacertideformus-specific qPCR assay. The qPCR diagnostic assay was run on the CFX96 (Bio-Rad) using the following thermocycling conditions: $95^{\circ} \mathrm{C}$ for 3 minutes, 40 cycles of $95^{\circ} \mathrm{C}$ for 10 seconds, and $56^{\circ} \mathrm{C}$ for 40 seconds. Each $10 \mu \mathrm{L}$ reaction consisted of $5 \mu \mathrm{L}$ of SensiFAST Probe No-ROX Kit (Bioline), $2 \mu \mathrm{L}$ of DNA $(5 \mathrm{ng}), 0.6 \mu \mathrm{L}$ of forward and reverse primers $(10 \mathrm{pmol} / \mu \mathrm{L}), 0.25 \mu \mathrm{L}$ probe $(10 \mathrm{pmol} / \mu \mathrm{L})$, and $2.15 \mu \mathrm{L}$ PCR water. A NTC (PCR water) and positive control (confirmed E. lacertideformus positive lesion) were included in the assay each time it was run. If oral swabs collected from a gecko at the completion of antibiotic treatment were negative via QPCR, the head, liver and/or diseased tissue fixed in alcohol from that animal were tested to confirm the accuracy of the negative result. A gecko was defined as qPCR positive for E. lacertideformus when one or more of the swabs or tissue samples tested positive.

\section{Histopathology}

The head and body of the formalin-fixed remains $(n=45)$ were individually sectioned mid-sagittally. Tissues were decalcified for 20 hours (Richard-Allan Scientific Decalcifying Solution, ThermoFisher Scientific), paraffin-embedded, sectioned at $4 \mu \mathrm{m}$, and stained with Haematoxylin and Eosin. Tissues were examined microscopically to (1) confirm the presence or absence of E. lacertideformus, and (2) determine the efficacy of administered antibiotics as assessed by the extent of $E$. lacertideformus infection and the immune response elicited. The severity of each lesion was scored from 0 to 4 (the percentage replacement of normal tissue volume); where $0=$ no lesion present, 1 = a mild lesion (1-25\%), 2 = a moderate lesion (25-50\%), $3=$ a severe lesion (51-74\%), and $4=$ an extensive lesion $(75-100 \%)$. 
The host inflammatory response was additionally scored on a scale from 0 to 4 (Fig. 1 ); where $0=$ no inflammation (Fig. $1 \mathrm{a}$ ), $1=$ mild inflammation adjacent to the bacterial colonies +/- perilesional cuffing and/or inflammatory infiltrate (Fig. 1b), 2 = moderate inflammation adjacent to the bacterial colonies +/- perilesional cuffing and/or inflammatory infiltrate (Fig. $1 \mathrm{c}$ ), $3=$ multifocal inflammatory infiltration into the lesion +/- evidence of fibroplasia (Fig. 1d), 4 = extensive and diffuse inflammatory infiltration into the lesion +/- evidence of fibroplasia (Fig. 1e). For each gecko, the total lesion score was calculated for all anatomical regions and grouped by the respective antibiotic treatment. A boxplot was generated from this data to compare the severity of histological lesions across different antibiotic treatments using the pandas library ${ }^{[13]}$ v. 1.2 .0 in Python v3.8 ${ }^{[11]}$.

A chi-square test was performed using the chisquare function from the Python library scipy.stats v1.5.4 ${ }^{[17]}$ to determine the efficacy of the antibiotics administered during the trial based on the inflammatory reaction observed in tissues. The antibiotic treatments enrofloxacin ( $\mathrm{n}=$ $4)$, amoxicillin clavulanic acid $(n=4)$, and the combination therapy $(n=3)$ (total, $n=11)$ were analysed against both the no treatment group $(n=8)$, and clarithromycin $(n=4)$, and rifampicin group $(n=4)$ (total, $n=16)$ to determine if they were significantly more effective. The clarithromycin and rifampicin antibiotic group were also analysed against the no treatment group. An inflammatory reaction of score $\geq 3$ was interpreted to indicate an appropriate host immune response, classifying the antibiotic as more effective. Inflammatory scores below this indicated a poor immune response. The antibiotics enrofloxacin, amoxicillin clavulanic acid, and the combination therapy were grouped because of their observed high efficacy based on the pronounced regression of lesion surface area, and minimal disease dissemination. Differences were considered statistically significant with $\mathrm{p} \leq 0.05$.

\section{Results}

gPCR optimisation, specificity and LOD

The qPCR primers and probe targeting a $171 \mathrm{bp}$ fragment of E. lacertideformus bacteria were successfully developed and optimised. The optimal final primer and probe concentrations were 600 and $250 \mathrm{nM}$, respectively. Amplification did not occur in the NTCs or when DNA from non-target bacterial species was tested.

A gBlock dilution equivalent to the detection of 122 copies/ $\mu \mathrm{L}$ was determined to be the limit of detection. The average Cq value for the LOD corresponded to a Cq and standard deviation of $36.95 \pm 0.16(\mathrm{COV}=0.45)$, respectively, with no Cq values produced in any of the replicates at dilution levels below this. The reaction efficiency $(E)$ and regression coefficient $\left(R^{2}\right)$ values were 92.39 and 0.99 , respectively.

\section{Confirmation of Enterococcus lacertideformus infection status}

All geckos assigned to the experimental inoculation groups (including co-housing treatment) and the control group prior to experimental challenge were confirmed negative for E. lacertideformus via qPCR of oral and skin swabs. All three geckos used for the collection of infected tissue for experimental inoculation were confirmed positive for E. lacertideformus by qPCR. Both affected geckos collected for the infection by contact trial were confirmed positive via qPCR in the samples collected prior to co-housing. The infection status of Asian house geckos experimentally inoculated with $E$. lacertideformus were confirmed by qPCR of oral and FNA swabs in animals grossly positive for $E$ lacertideformus, and via qPCR of oral swabs and tissue samples, and histology in animals grossly negative for E. lacertideformus (Table 3). Prior to enlistment into the antibiotic treatment trial, all geckos confirmed grossly infected with E. lacertideformus had a positive oral swab and/or FNA swab by qPCR (Table 3). All naturally infected geckos used in the treatment trial were positive for E. lacertideformus via qPCR.

\section{Clinical course of Enterococcus lacertideformus}

The clinical course of experimentally and naturally infected geckos is summarised in Supplementary Table S1. Of the animals that developed disease, geckos infected via the skin laceration and mucosal abrasion routes had the shortest incubation periods, averaging 54 ( 47 to 58 days, $n=5$ ) and 74 (69 to 78 days, $n=3$ ) days, respectively. Geckos inoculated via coelomic injection and by mouth averaged longer incubation periods of 99 (98 to 101 days, $n=3$ ) and 102 (101 to 103 days, $n=2$ ) days, respectively. Geckos in the subcutaneous injection and co-housing groups did not develop gross lesions (Figure 2).

\section{Macroscopic lesions of geckos infected with E. lacertideformus}

No macroscopic lesions were seen in geckos infected with E. lacertideformus through the subcutaneous route. When geckos were inoculated by the skin laceration route, lesions developed at the site of inoculation in three of five geckos, and in two of five geckos lesions developed in the cheek and the maxillary mucosa or perivascular tissues surrounding the eye (Supplementary Table S2). When the inoculum was administered directly by mouth, gross lesions in the oral mucosa of the mandible or maxilla developed in two of five geckos. Abrasion of the oral mucosa resulted in lesions in three of five geckos in the mandibular mucosa. The coelomic cavity injection resulted in a single ventral

Page $7 / 20$ 
abdominal mass in three of five geckos. The skin laceration route was the only inoculation method to produce macroscopic lesions in all geckos. The distribution of the macroscopic lesions observed in the viscera of the infected geckos is shown in Figure 3 . Gross bacterial colonies were found in the kidney $(n=9)$, liver $(n=6)$, pancreas $(n=3)$, lung $(n=2)$, stomach $(n=1)$, and colon $(n=1)$ of E. lacertideformus infected geckos (Supplementary Table S2).

\section{Change in the surface area of macroscopic lesions following treatment}

Geckos given enrofloxacin ( $n=4)$, amoxicillin clavulanic acid $(n=4)$, and the combination therapy $(n=3)$ had the greatest reduction in lesion surface area over the treatment period (Figure 4), ranging from 54.7 to 100.0 percent (Figure 5). When geckos were given clarithromycin ( $n=4)$ and rifampicin $(n=4)$, the reduction in the lesion surface area ranged from 25.0 to 66.7 percent, with the exception of a single gecko given clarithromycin which had an increase in the surface area of the lesion by 33.3 percent (Figure 5). The surface area of lesions in geckos given enrofloxacin, the combination therapy, amoxicillin clavulanic acid, rifampicin, and clarithromycin changed by an average of -83.6 (range: -100.0 to -70.7 ), -62.4 (range: -66.6 to -56.7 ), -58.2 (range: -65.0 to -54.7 ), -45.5 (range: -66.7 to -25.0 ), and -26.5 (range: -60.0 to +33.3 ) percent, respectively (Supplementary Table S2).

Three geckos inoculated by mucosal abrasion $(n=3)$ and 1 inoculated by coelomic cavity injection $(n=1)$ were not included in the treatment trial due to logistical limitations experienced during the trial. Macroscopic lesions of these experimentally infected geckos not treated with antibiotics continued to enlarge until they were euthanised at 28 days (Supplementary Table S2). Over the course of disease, the surface area of the lesion at the inoculation site in untreated geckos in the mucosal abrasion group increased between 233.3 and 300.0 percent. The lesion surface area of the single untreated gecko in the coelomic cavity group increased 100.0 percent.

\section{Distribution of lesions as a function of route of inoculation}

Evidence of E. lacertideformus infection was observed in the tissues of 17 experimentally infected geckos (Supplementary Table S3). Irrespective of the antibiotic treatment status, geckos inoculated via the skin laceration route were observed to have a more extensive spread of disease from the original inoculation site (Figure 6). Lesions in geckos inoculated via the oral cavity, mucosal abrasion, and subcutaneous injection were confined to the head and neck (Figure 6). Dissemination of E. lacertideformus in geckos inoculated via the coelomic route and treated with antibiotics were confined to the liver, pericardium, pleura, and injection site (coelomic cavity), however, the bacteria were observed to have disseminated to the parenchyma of the lungs and heart in one gecko not treated with antibiotics. The severity of the bacterial lesions across all groups was most pronounced in the skin laceration group, in which the highest disease grade (grade 4) was documented in two of the five geckos inoculated (Figure 6).

\section{Distribution of lesions in naturally infected geckos}

Evidence of E. lacertideformus was observed in the tissues of 10 geckos infected naturally (Supplementary Table S3). All geckos infected naturally had histological lesions in one or more parts of the head, particularly at the crown of the head, within the mandible, and the region surrounding the eyes. Most naturally infected geckos had dissemination of E. lacertideformus organisms to internal organs/tissues (60\%, $\mathrm{n}$ $=6)$, particularly to the lungs $(50 \%, n=5)$ and kidney $(40 \%, n=4)$. Irrespective of the antibiotic, geckos with advanced disease (i.e. spread to multiple internal organs/tissues) typically had higher lesion severity scores than geckos with disease confined entirely to the head (Figure 6).

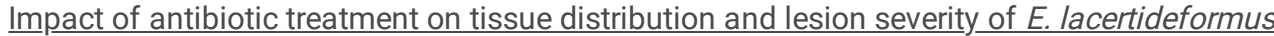

Five geckos inoculated by the skin laceration method were treated with clarithromycin $(n=2)$, rifampicin $(n=1)$, amoxicillin clavulanic acid $(n=1)$, and enrofloxacin $(n=1)$. Geckos treated with enrofloxacin and amoxicillin clavulanic acid had reduced lesion severity scores as compared to those treated with clarithromycin or rifampicin (Figure 7). Additionally, dissemination of disease to viscera did not occur in geckos treated with amoxicillin or enrofloxacin but dissemination did occur in the two animals treated with clarithromycin and the one animal treated with rifampicin (Figure 6). Overall, the histological score of lesions for naturally and experimentally infected geckos that were treated with clarithromycin and rifampicin, and geckos that were not treated with antibiotics were higher than for geckos given enrofloxacin, amoxicillin clavulanic acid, and the combined therapy (Figure 6).

\section{Histological inflammatory response}

Histologically, if inflammation was present, the predominant leukocyte associated with the presence of E. lacertideformus lesions were lymphocytes (Figure 1; Supplementary Table S4). Scattered heterophils admixed with the lymphocytes were common, and histiocytes and multinucleate giant cells were observed less frequently. An inflammatory response to E. lacertideformus was absent in five of eight geckos that were not treated, the remaining two geckos had a mild inflammatory response (Inflammation score $=1$ ), and a single gecko had a

Page $8 / 20$ 
moderate inflammatory response (Inflammation scores ranging from 2 to 3 ). A mild inflammatory response was observed in all geckos administered clarithromycin and rifampicin (Figure 7), however, a single gecko given rifampicin had a mild to moderate inflammatory response (Inflammation scores ranging from 2 to 3 ). In experimentally and naturally infected geckos, the most pronounced host inflammatory responses (Inflammation scores ranging from 3 to 4), predominantly characterised by infiltration of immune cells, destruction of bacterial colonies, and fibroplasia were associated with geckos administered enrofloxacin, amoxicillin clavulanic acid, and the enrofloxacin and amoxicillin clavulanic acid combination therapy (Figure 7; Supplementary Table S4). A significant difference with respect to inflammatory response was observed for these three antibiotics when analysed against clarithromycin, and rifampicin $(p=0.001)$, and geckos untreated with antibiotics $(p=0.001)$. Comparison of the no treatment group to clarithromycin and rifampicin antibiotics was also significantly different $(p=0.005)$.

\section{Confirmation of Enterococcus lacertideformus infection status at euthanasia}

The infection status of all treated Asian house geckos at treatment completion or euthanasia was confirmed via qPCR of oral swabs and/or tissues, and histology (Table 4). Following 21 days of treatment, all geckos were confirmed infected with E. lacertideformus by histology and qPCR (Table 4). At trial completion all members of the uninfected control group were negative for $E$. lacertideformus via all detection means. Both naturally affected geckos caught for the co-housing trial were positive for E. lacertideformus via qPCR at euthanasia.

\section{Discussion}

The first objective of this study was to determine if Asian house geckos could be experimentally infected with E. lacertideformus. This study showed that disease closely resembling that seen in geckos naturally infected with E. lacertideformus can be experimentally induced by application of a suspension of this bacterium to lacerated skin or abraded oral mucosa, by subcutaneous injection, and by oral administration. Intraperitoneal injection with E. lacertideformus was also able to induce an infection, however, the lesions were confined to the coelomic cavity. Although only small numbers of animals were used in this study, it appears that inoculating a skin laceration will most consistently result in infection and disease, and the time between inoculation and the onset of gross lesions will be shortest. Also, this was the only route of inoculation that resulted in dissemination from the original inoculation site, most similar to natural infection. This study additionally showed that infection can be transmitted between geckos by co-housing them, although only oral colonisation was demonstrated, and disease did not develop over the course of the trial.

These findings provide insight into the possible mechanisms of $E$. lacertideformus transmission between infected and uninfected reptiles. Asian house geckos and blue-tailed skinks are known to bite each other during fighting and mating ${ }^{[18,19]}$, and cannibalism in Asian house geckos has been documented ${ }^{[19]}$. Biting also occurs as male blue-tailed skinks subdue other male blue-tailed skinks in same sex sexual encounters. In both these instances, if the biting lizard had an oral infection with E. lacertideformus and is able to penetrate the skin of the lizard they are biting, then this natural behaviour effectively replicates the skin laceration inoculation group in this study. Cannibalism of an infected gecko by an uninfected gecko could also potentially result in infection if bacteria were released from a head or a coelomic lesion when the animal was being consumed.

How E. lacertideformus is transmitted between the infected geckos and co-housed geckos in this study is not known. No evidence of aggression, including bite wounds was observed during co-housing, however, geckos were not monitored continuously, and therefore aggression may have occurred. Another possible route of transmission would be that of environmental contamination by the infected gecko. Both geckos likely drank water from the same source. Also, while not yet studied, based on previous histological evidence of oral and gastrointestinal lesions shedding organisms, it is possible that $E$. lacertideformus could be shed in faeces resulting in faecal-oral contamination. Both geckos infected during the co-housing trial had oral colonisation by $E$. lacertideformus but did not develop disease during the experimental period. This finding is consistent with the observation that the geckos experimentally infected with the oral inoculation route took the longest to develop disease.

The second objective of this study was to determine if antibiotic treatment could be used successfully to cure infected geckos. The antibiotic treatments administered during the trial were chosen based on one or more of the following criteria: efficacy against Gram positive bacteria, ability to penetrate biofilms, therapeutic index, previous use in reptiles, capacity to administer via the oral route, and susceptibility to $E$. lacertideformus and other species of enterococci. A screen of the $E$. lacertideformus genome for antimicrobial resistance genes revealed a resistance profile only to the antibiotics trimethoprim, tetracycline, streptothricin, and bacitracin ${ }^{[7]}$.

All five treatment protocols used in this study caused a reduction in the size of gross lesions. However, it appears that enrofloxacin would be the drug of choice for treating $E$. lacertideformus in infected reptiles as the geckos treated with it had the largest reduction in the size of their gross lesions, the lowest average lesion scores, and were more likely to have lesions containing an inflammatory response, possibly in 
response to dying bacteria. Amoxicillin clavulanic acid also showed a similar but more limited impact on E. lacertideformus induced lesions and would be a second choice for treatment if enrofloxacin was not available. Combination therapy using both enrofloxacin and amoxicillin clavulanic acid did not appear to improve the treatment outcome.

Despite evidence of the bactericidal activity and biofilm penetrating capability of enrofloxacin ${ }^{[20]}$ and amoxicillin clavulanic acid treatments $[21,22]$, all but one of the geckos, an enrofloxacin treated animal, were shown to remain grossly infected with E. lacertideformus at the end of the 21-day treatment period. Therefore, it is unclear whether antimicrobial treatment will completely clear an animal of infection with longer treatment periods, or treatment periods with an increased dose or dosage frequency. The geckos in this study continued to eat and maintain their weight during the treatment period, so a longer duration of treatment would appear to be a safe option. Single-dose oral administration of enrofloxacin in Asian house geckos has been shown to reach concentrations that exceed the minimum inhibitory concentrations that would be effective for other enterococcal species when given at the dosage rate $(10 \mathrm{mg} / \mathrm{kg})$ used in this study ${ }^{[23]}$. However, as $E$. lacertideformus produces a biofilm, and biofilm producing bacteria often require an antimicrobial MIC up to 100 times higher than planktonic bacteria ${ }^{[24,25]}$, the dosage rate and frequency would likely need to be increased. Increasing the dosage rate or frequency would lead to increased blood and likely tissue concentrations of the antibiotic, and these increased concentrations might be more effective at penetrating the biofilm and achieving the therapeutic range needed to eliminate $E$. lacertideformus. However, this would not be recommended unless additional pharmacokinetic studies were undertaken to ensure that drug concentrations did not become toxic.

\section{Conclusion}

The Asian house gecko represents a robust model to study the dynamics of $E$. lacertideformus disease. This gecko is a highly invasive species of similar mass, physiology, and preferred habitat to the critically endangered Listers gecko and blue-tailed skink on Christmas Island. Active monitoring of reptiles affected with E. lacertideformus and the development of treatment protocols proves imperative as multisystemic spread of the organism throughout the body and ultimate death have been documented in all cases of untreated lizards ${ }^{[2]}$. This research showed that infection of geckos with E. lacertideformus can be established experimentally via inoculation, and naturally by means of direct contact through co-housing. However, inoculation of a skin wound appears to result in the highest infection rate, with the shortest incubation period and greatest chance of dissemination to the coelomic viscera, most closely mimicking natural infection. Due to frequent colonisation of the oral cavity by $E$. lacertideformus in experimentally and naturally infected geckos, the molecular analysis of oral swabs collected from wild or captively housed reptiles using the developed $E$. lacertideformus-specific qPCR may serve as a non-invasive and reliable diagnostic tool and disease surveillance method. When geckos infected with $E$. lacertideformus were treated with antibiotics all geckos exhibited some degree of lesion regression, though cure was not achieved in any case. Enterococcus lacertideformus appears to be sensitive to enrofloxacin, and possibly amoxicillin clavulanic acid, in-vitro, but treatment regiments more than 21 days will be required to achieve a cure at the dosage rates administered in this study. Further research into the pharmacology of amoxicillin clavulanic acid, and enrofloxacin in Asian house geckos will be required to define the appropriate dose, dose frequency, and period of treatment required to achieve the therapeutic range needed to eliminate E. lacertideformus. Knowledge of the drugs MIC may lead to the development of novel and highly effective therapeutic and prophylactic treatment protocols necessary to protect susceptible reptiles.

\section{Declarations}

\section{Data Availability}

Most data generated and analysed during this study are included in this published article (and its Supplementary Information files). Other datasets are available from the corresponding author on reasonable request.

\section{Acknowledgements}

The authors thank Christmas Island National Park, Parks Australia, the Sydney School of Veterinary Science, University of Sydney, Taronga Conservation Society Australia, and Taronga Conservation Science Initiative for their logistical and financial support. Further financial support for this project was provided by the Australia and Pacific Science Foundation [grant number APSF 17/6], the Holsworth Wildlife Research Endowment - Equity Trustees Charitable Foundation and Ecological Society of Australia [grant number G200647], and the National Environmental Science Programme - Threatened Species Recovery Hub [grant number NESP 2.3.5]. The authors also thank Christopher Dryburgh, Felicity Evans, Brendan Tiernan and Kristen Schubert for their volunteer efforts, and Jane Hall and Paul Thompson from the Australian Registry of Wildlife Health, Taronga Zoo for their logistical and scientific support.

\section{Author Contributions}


ORCID iD: 0000-0001-8735-1222

Contributor role: Conceptualisation, methodology, software, validation, formal analysis, investigation, resources, data curation, writing original draft preparation, writing - review and editing, visualisation, project administration, funding acquisition.

\section{Karrie Rose}

ORCID iD: 0000-0001-9828-538X

Contributor role: Conceptualisation, methodology, visualisation, writing - review and editing, supervision, project administration, funding acquisition.

\section{John-Paul Emery}

ORCID iD: 0000-0001-9984-2504

Contributor role: Investigation, resources, data curation, writing - review and editing.

\section{David Phalen}

ORCID iD: 0000-0003-2387-4061

Contributor role: Conceptualisation, methodology, investigation, visualisation, resources, writing - review and editing, supervision, project administration, funding acquisition.

\section{Competing Interests Statement}

The authors declare no competing interests.

\section{References}

1. IUCN. The IUCN Red List of Threatened Species, <https://www.iucnredlist.org/resources/summary-statistics> (2019).

2. Cogger, H., Sadlier, R. \& Cameron, E. The Terrestrial Reptiles of Australia's Island Territories(Australian National Parks and Wildlife Service, 1983).

3. Yang, B. et al. Combination Susceptibility Testing of Common Antimicrobials in Vitro and the Effects of Sub-MIC of Antimicrobials on Staphylococcus aureus Biofilm Formation. Frontiers in microbiology. 8, 2125-2125 https://doi.org/10.3389/fmicb.2017.02125 (2017).

4. Sedlacek, M. J. \& Walker, C. Antibiotic resistance in an in vitro subgingival biofilm model. Oral Microbiol Immunol. 22, 333-339 https://doi.org/10.1111/j.1399-302X.2007.00366.x (2007).

5. Božić, D. D. et al. Antibiofilm effects of amoxicillin-clavulanic acid and levofloxacin in patients with chronic rhinosinusitis with nasal polyposis. European Archives of Oto-Rhino-Laryngology. 275, 2051-2059 https://doi.org/10.1007/s00405-018-5049-6 (2018).

6. Agius, J. et al. Pharmacokinetic profile of enrofloxacin and its metabolite ciprofloxacin in Asian house geckos (Hemidactylus frenatus) after single-dose oral administration of enrofloxacin. Veterinary and Animal Science. 9, 100116 https://doi.org/10.1016/j.vas.2020.100116 (2020).

7. Girard, L. P., Ceri, H., Gibb, A. P., Olson, M. \& Sepandj, F. MIC versus MBEC to determine the antibiotic sensitivity of Staphylococcus aureus in peritoneal dialysis peritonitis. Perit Dial Int. 30, 652-656 https://doi.org/10.3747/pdi.2010.00010 (2010).

8. Di Domenico, E. G. et al. Inflammatory cytokines and biofilm production sustain Staphylococcus aureus outgrowth and persistence: a pivotal interplay in the pathogenesis of Atopic Dermatitis. Sci Rep. 8, 9573-9573 https://doi.org/10.1038/s41598-018-27421-1 (2018).

9. Manner, S., Goeres, D. M., Skogman, M., Vuorela, P. \& Fallarero, A. Prevention of Staphylococcus aureus biofilm formation by antibiotics in 96-Microtiter Well Plates and Drip Flow Reactors: critical factors influencing outcomes. Sci Rep 7, 43854, doi:10.1038/srep43854 (2017).

10. Percie du Sert, N. et al. The ARRIVE guidelines 2.0: Updated guidelines for reporting animal research. PLOS Biology 18, e3000410, doi:10.1371/journal.pbio.3000410 (2020).

11. van Rossum, G. Python tutorial. (Centrum voor Wiskunde en Informatica (CWI), Amsterdam, 1995).

12. Rose, K. Wildlife Health Investigation Manual. (The Australian Registry of Wildlife Health, 2007).

13. pandas-dev/pandas: Pandas v. 1.2 .0 (Zenodo, 2020). 
14. Ye, J. et al. Primer-BLAST: a tool to design target-specific primers for polymerase chain reaction. BMC Bioinformatics 13, 134, doi:10.1186/1471-2105-13-134 (2012).

15. Benson, D. A. et al. GenBank. Nucleic Acids Res 41, D36-42, doi:10.1093/nar/gks1195 (2013).

16. Altschul, S. F., Gish, W., Miller, W., Myers, E. W. \& Lipman, D. J. Basic local alignment search tool. Journal of Molecular Biology 215, 403410, doi:https://doi.org/10.1016/S0022-2836(05)80360-2 (1990).

17. Virtanen, P. et al. SciPy 1.0: fundamental algorithms for scientific computing in Python. Nature Methods 17, 261-272, doi:10.1038/s41592-019-0686-2 (2020).

18. Yuki, M. \& Fumio, H. Behavioral Interactions for Food among Two Clones of Parthenogenetic $<\mathrm{i}>$ Lepidodactylus lugubris $</ \mathrm{i}>$ and Sexually Reproducing < i > Hemidactylus frenatus</i > Geckos. Current Herpetology 37, 124-132, doi:10.5358/hsj.37.124 (2018).

19. Cogger, H., Sadlier, R. \& Cameron, E. The Terrestrial Reptiles of Australia's Island Territories. (Australian National Parks and Wildlife Service, 1983).

20. Yang, B. et al. Combination Susceptibility Testing of Common Antimicrobials in Vitro and the Effects of Sub-MIC of Antimicrobials on Staphylococcus aureus Biofilm Formation. Frontiers in microbiology 8, 2125-2125, doi:10.3389/fmicb.2017.02125 (2017).

21. Sedlacek, M. J. \& Walker, C. Antibiotic resistance in an in vitro subgingival biofilm model. Oral Microbiol Immuno/ 22, 333-339, doi:10.1111/j.1399-302X.2007.00366.x (2007).

22. Božić, D. D. et al. Antibiofilm effects of amoxicillin-clavulanic acid and levofloxacin in patients with chronic rhinosinusitis with nasal polyposis. European Archives of Oto-Rhino-Laryngology 275, 2051-2059, doi:10.1007/s00405-018-5049-6 (2018).

23. Agius, J. et al. Pharmacokinetic profile of enrofloxacin and its metabolite ciprofloxacin in Asian house geckos (Hemidactylus frenatus) after single-dose oral administration of enrofloxacin. Veterinary and Animal Science 9, 100116, doi:https://doi.org/10.1016/j.vas.2020.100116 (2020).

24. Girard, L. P., Ceri, H., Gibb, A. P., Olson, M. \& Sepandj, F. MIC versus MBEC to determine the antibiotic sensitivity of Staphylococcus aureus in peritoneal dialysis peritonitis. Perit Dial Int 30, 652-656, doi:10.3747/pdi.2010.00010 (2010).

25. Di Domenico, E. G. et al. Inflammatory cytokines and biofilm production sustain Staphylococcus aureus outgrowth and persistence: a pivotal interplay in the pathogenesis of Atopic Dermatitis. Sci Rep 8, 9573-9573, doi:10.1038/s41598-018-27421-1 (2018).

\section{Tables}

Table 1: The treatment groups used in the experimental infection trial.

\begin{tabular}{|c|c|c|c|c|}
\hline Treatment & Route & $\begin{array}{l}\text { Bacterial } \\
\text { Dose* }\end{array}$ & $\begin{array}{l}\text { Inoculum } \\
\text { Volume } \\
\text { (mL) }\end{array}$ & Site of Inoculation \\
\hline Control & SC & 0 & 0.02 & $\begin{array}{l}\text { Saline injection into the loose skin } 2 \mathrm{~mm} \text { off the ventral midline, and } 5 \mathrm{~mm} \text { cranial to } \\
\text { the pelvis. }\end{array}$ \\
\hline Oral cavity & PO & 80,000 & 0.02 & Direct administration into the oral cavity. \\
\hline Subcutaneous & SC & 80,000 & 0.02 & Subcutaneous injection into the loose skin at the medial aspect of the neck. \\
\hline $\begin{array}{l}\text { Mucosal } \\
\text { abrasion }\end{array}$ & & 80,000 & 0.02 & $\begin{array}{l}\text { Shallow abrasion of the gingiva on the right side of the face using a cotton swab. } \\
\text { Inoculum applied to abraded surface. }\end{array}$ \\
\hline $\begin{array}{l}\text { Skin } \\
\text { laceration }\end{array}$ & & 80,000 & 0.02 & $\begin{array}{l}\text { Shallow skin laceration ( } 1 \mathrm{~mm} \text { deep and } 5 \mathrm{~mm} \text { long) on the right lateral side of the } \\
\text { face rostral to the ear ostium. Inoculum applied to wound surface. Wounds covered } \\
\text { by a light-weight adhesive bandage for } 24 \text { hours. }\end{array}$ \\
\hline $\begin{array}{l}\text { Coelomic } \\
\text { cavity }\end{array}$ & IC & 80,000 & 0.02 & Co-housed with an infected animal for ten days. \\
\hline Co-housing & & $\mathrm{n} / \mathrm{a}$ & $\mathrm{n} / \mathrm{a}$ & Natural infection means. \\
\hline
\end{tabular}

SC, subcutaneous; PO, per os; IC, intracoelomic; * total number of organisms administered per injection.

Table 2: Primer and probe set used in the Enterococcus lacertideformus-specific qPCR assay. 


\begin{tabular}{|llllll|}
\hline Sequence ID* & $\mathbf{5}^{\mathbf{}}$ à 3'Sequence $^{\prime}$ & Length $(\mathbf{b p})$ & $\mathbf{T m}\left({ }^{\circ} \mathbf{C}\right)$ & $\mathbf{G C}(\%)$ & Amplicon Length (bp) \\
\hline EntL - Forward & CCAAATAATAGATGCGATTCCC & 22 & 59 & 40.9 & 171 \\
\hline EntL - Reverse & CTACTATCCAGTCACTCAATCC & 22 & 59 & 45.5 & \\
\hline EntL - Probe & TGGGTTGAATCATTGACATCGTGAGA & 26 & 66 & 42 & \\
\hline
\end{tabular}

Table 3: Prevalence of gross lesions and infection status of Asian house geckos experimentally challenged with E. lacertideformus by six different inoculation methods.

\begin{tabular}{|c|c|c|c|c|c|c|c|c|c|c|c|c|}
\hline \multirow{4}{*}{$\begin{array}{l}\text { Treatment } \\
\text { Group }\end{array}$} & \multirow{3}{*}{\multicolumn{2}{|c|}{$\begin{array}{l}\text { Gross Disease } \\
\text { Status } \square\end{array}$}} & \multirow{2}{*}{\multicolumn{4}{|c|}{$\begin{array}{l}\text { Gross Disease Positive } \\
\text { Disease Status - qPCR* }\end{array}$}} & \multicolumn{6}{|c|}{ Gross Disease Negative } \\
\hline & & & & & & & \multicolumn{4}{|c|}{ Disease Status - qPCR* } & \multirow{2}{*}{\multicolumn{2}{|c|}{ Histology }} \\
\hline & & & \multicolumn{2}{|c|}{ Oral Swab } & \multicolumn{2}{|c|}{ FNA Lesion } & \multicolumn{2}{|c|}{ Oral Swab } & \multicolumn{2}{|l|}{ Tissue } & & \\
\hline & $+(\%)$ & $-(\%)$ & $+(\%)$ & $-(\%)$ & $+(\%)$ & $-(\%)$ & $+(\%)$ & $-(\%)$ & $+(\%)$ & $-(\%)$ & $+(\%)$ & $-(\%)$ \\
\hline Oral cavity & $\begin{array}{l}2 \\
(40 \%)\end{array}$ & $\begin{array}{l}3 \\
(60 \%)\end{array}$ & $\begin{array}{l}2 \\
(100 \%)\end{array}$ & $0(0 \%)$ & $\begin{array}{l}2 \\
(100 \%)\end{array}$ & $\begin{array}{l}0(0 \\
\%)\end{array}$ & $0(0 \%)$ & $\begin{array}{l}3 \\
(100 \%)\end{array}$ & $0(0 \%)$ & $\begin{array}{l}3 \\
(100 \%)\end{array}$ & $\begin{array}{l}0(0 \\
\%)\end{array}$ & $\begin{array}{l}3 \\
(100 \%)\end{array}$ \\
\hline Subcutaneous & $0(0 \%)$ & $\begin{array}{l}5 \\
(100 \%)\end{array}$ & $n / a$ & $n / a$ & $n / a$ & $\mathrm{n} / \mathrm{a}$ & $0(0 \%)$ & $\begin{array}{l}5 \\
(100 \%)\end{array}$ & $\begin{array}{l}3 \\
(60 \%)\end{array}$ & $\begin{array}{l}2 \\
(40 \%)\end{array}$ & $\begin{array}{l}3 \\
(60 \%)\end{array}$ & $\begin{array}{l}2 \\
(40 \%)\end{array}$ \\
\hline $\begin{array}{l}\text { Mucosal } \\
\text { abrasion }\end{array}$ & $\begin{array}{l}3 \\
(60 \%) \\
\ddagger\end{array}$ & $\begin{array}{l}2 \\
(40 \%)\end{array}$ & $\begin{array}{l}3 \\
(100 \%)\end{array}$ & $0(0 \%)$ & 3 & $\begin{array}{l}0 \\
(0 \%)\end{array}$ & 2 & $0(0 \%)$ & $\begin{array}{l}2 \\
(100 \%)\end{array}$ & $0(0 \%)$ & $\begin{array}{l}1 \\
(50 \%)\end{array}$ & $\begin{array}{l}1(50 \\
\%)\end{array}$ \\
\hline $\begin{array}{l}\text { Skin } \\
\text { laceration }\end{array}$ & $\begin{array}{l}5 \\
(100 \%)\end{array}$ & $0(0 \%)$ & $\begin{array}{l}1 \\
(20 \%)\end{array}$ & $\begin{array}{l}4 \\
(80 \%)\end{array}$ & $\begin{array}{l}5 \\
(100 \%)\end{array}$ & $\begin{array}{l}0 \\
(0 \%)\end{array}$ & $n / a$ & $\mathrm{n} / \mathrm{a}$ & $\mathrm{n} / \mathrm{a}$ & $n / a$ & $\mathrm{n} / \mathrm{a}$ & $n / a$ \\
\hline $\begin{array}{l}\text { Coelomic } \\
\text { cavity }\end{array}$ & $\begin{array}{l}3 \\
(60 \%) \\
\ddagger\end{array}$ & $\begin{array}{l}2 \\
(40 \%)\end{array}$ & $0(0 \%)$ & $\begin{array}{l}3 \\
(100 \%)\end{array}$ & $\begin{array}{l}3 \\
(100 \%)\end{array}$ & $\begin{array}{l}0 \\
(0 \%)\end{array}$ & $0(0 \%)$ & $\begin{array}{l}2 \\
(100 \%)\end{array}$ & $0(0 \%)$ & $\begin{array}{l}2 \\
(100 \%)\end{array}$ & $\begin{array}{l}0(0 \\
\%)\end{array}$ & $\begin{array}{l}2 \\
(100 \%)\end{array}$ \\
\hline Co-housing & $0(0 \%)$ & $\begin{array}{l}5 \\
(100 \%)\end{array}$ & $n / a$ & $n / a$ & $n / a$ & $n / a$ & $\begin{array}{l}2 \\
(40 \%)\end{array}$ & $\begin{array}{l}3 \\
(60 \%)\end{array}$ & $\begin{array}{l}2 \\
(40 \%)\end{array}$ & $\begin{array}{l}3 \\
(60 \%)\end{array}$ & $\begin{array}{l}0(0 \\
\%)\end{array}$ & $\begin{array}{l}5 \\
(100 \%)\end{array}$ \\
\hline
\end{tabular}

Q, A gecko was defined as grossly positive for E. lacertideformus when an FNA cytology of the lesion demonstrated characteristic organisms.

*, A gecko was defined as QPCR positive for E. lacertideformus when the oral swab and/or FNA of the gross lesion tested returned a positive result. ¥, A gecko grossly positive for $E$. lacertideformus infection but not included in the antibiotic treatment trial (mucosal abrasion, $n=3$; coelomic cavity, $n=1$ ).

All remaining geckos confirmed grossly positive for E. lacertideformus were enlisted into the antibiotic treatment trial.

Table 4: Infection status of Asian house geckos given antibiotics at the end of the treatment period.

\begin{tabular}{|lllllllll|}
\hline Treatment Group & \multicolumn{2}{l}{ Disease Status } & \multicolumn{2}{l}{ Disease Status - qPCR* } & \multicolumn{3}{c|}{ Histology口 } \\
\cline { 2 - 8 } & Gross & & Oral Swab & Tissue & & \\
\cline { 2 - 8 } & $+(\%)$ & $-(\%)$ & $+(\%)$ & $-(\%)$ & $+(\%)$ & $-(\%)$ & $+(\%)$ & $-(\%)$ \\
\hline Oral cavity & $2(100 \%)$ & $0(0 \%)$ & $2(100 \%)$ & $0(0 \%)$ & $2(100 \%)$ & $0(0 \%)$ & $2(100 \%)$ & $0(0 \%)$ \\
\hline Skin laceration & $5(100 \%)$ & $0(0 \%)$ & $1(20 \%)$ & $4(80 \%)$ & $5(100 \%)$ & $0(0 \%)$ & $5(100 \%)$ & $0(0 \%)$ \\
\hline Coelomic cavity & $2(100 \%)$ & $0(0 \%)$ & $0(0 \%)$ & $2(100 \%)$ & $2(100 \%)$ & $0(0 \%)$ & $2(100 \%)$ & $0(0 \%)$ \\
\hline Naturally infected & $9(90 \%)$ & $1(10 \%)$ & 10 & $0(0 \%)$ & $10(100 \%)$ & $0(0 \%)$ & $10(100 \%)$ & $0(0 \%)$ \\
\hline
\end{tabular}

*, A gecko was defined as qPCR positive for E. lacertideformus when the oral swab and/or tissue samples returned a positive result.

\, A gecko was defined as histologically positive for E. lacertideformus when characteristic organisms were observed in the tissues.

Figures 

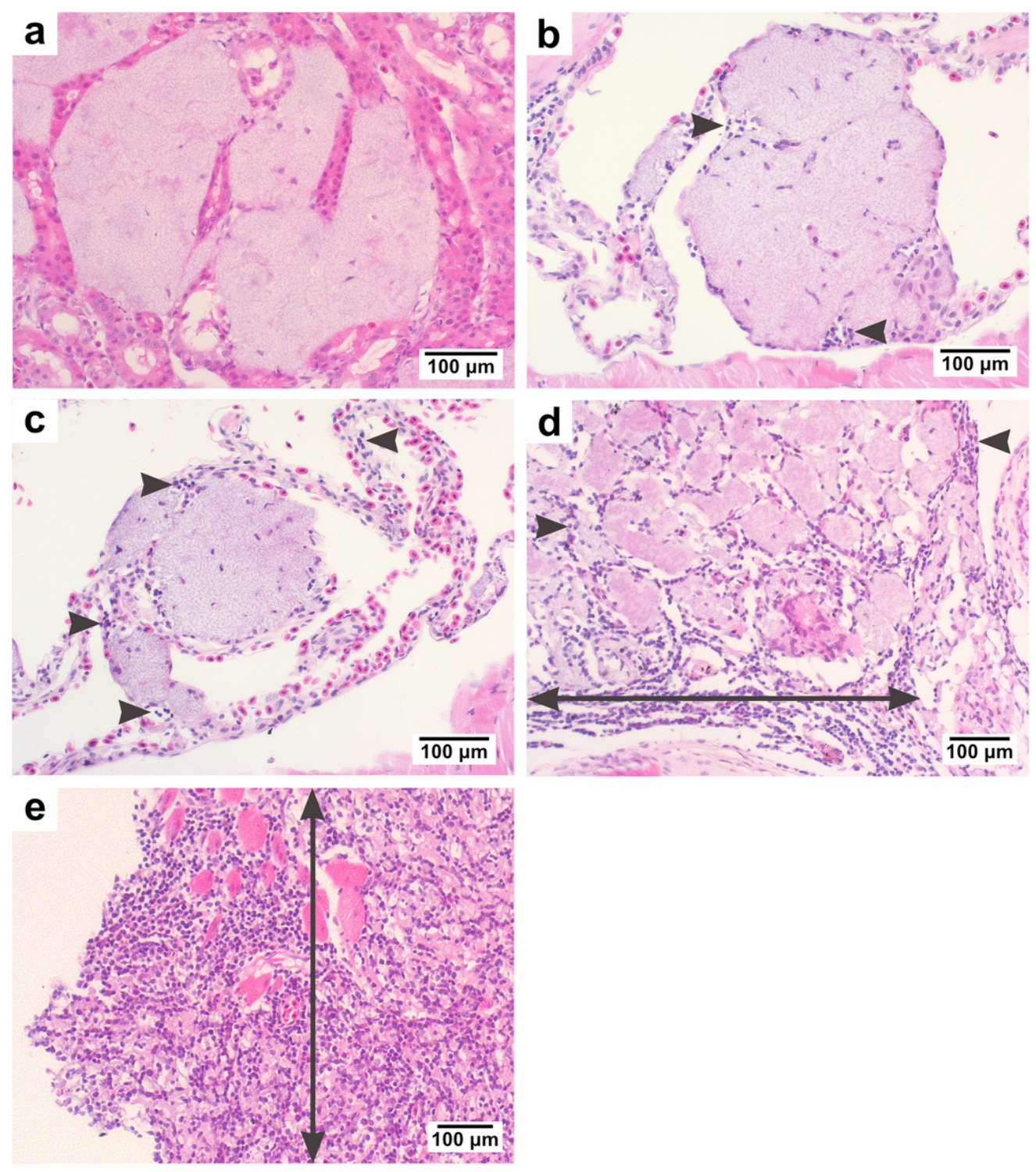

\section{Figure 1}

Histological sections stained with Haematoxylin and Eosin showing the range of inflammatory severity in Asian house geckos infected with E. lacertideformus. (a) Coalescing colonies of E. lacertideformus within the kidney associated with no inflammation (inflammatory score = 0), (b) large focal colony of E. lacertideformus in the lung containing mild inflammation adjacent to the bacterial colonies with perilesional cuffing (inflammatory score $=1$, grey arrowheads), (c) focal colony of E. lacertideformus in the liver containing moderate inflammation adjacent to the bacterial colonies with perilesional cuffing and minimal inflammatory infiltrate (inflammatory score $=2$, grey arrowheads), (d) multifocal colonies of E. lacertideformus markedly distending the soft tissues of the mandible and containing multifocal to coalescing inflammatory infiltrate with evidence of fibroplasia (inflammatory score $=3$, double pointed arrow demonstrates location and range of inflammation), (e) dispersed colonies of E. lacertideformus replacing the soft tissue of the buccal subcutis or lamina propria and containing extensive and diffuse inflammatory infiltrates with evidence of fibroplasia (inflammatory score $=4$, grey arrowheads, double pointed arrow demonstrates the location and range of inflammation). 


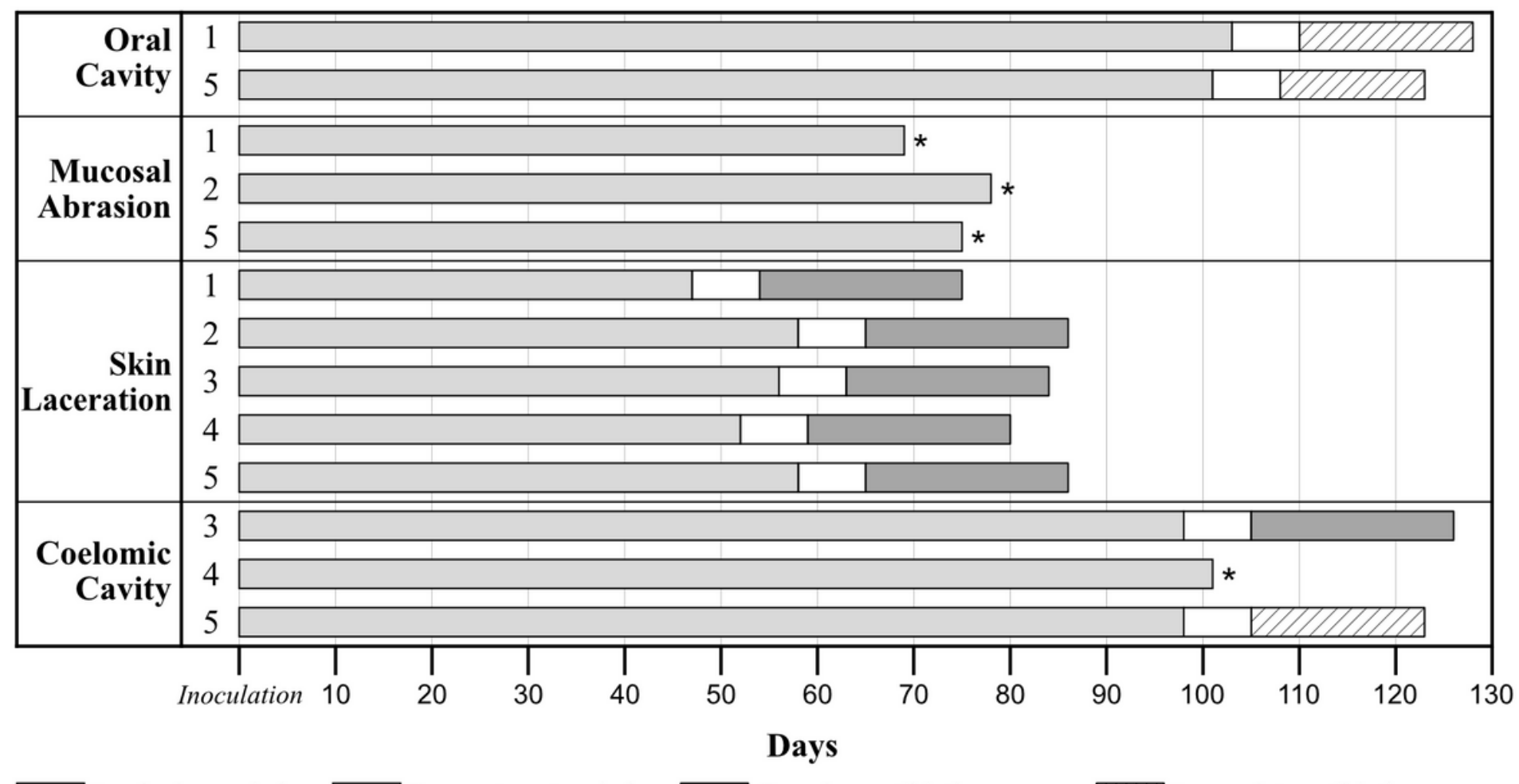

Incubation period $\square$ Pre-treatment period

Complete antibiotic course

ZII Incomplete antibiotic course

Figure 2

Bar chart illustrating the incubation period, pre-treatment period (period from confirmation of infection and onset of treatment), and treatment duration as a function of route of E. lacertideformus inoculation. White, light grey, dark grey, and hatch represent the pre-treatment period, incubation period, complete antibiotic course, and incomplete antibiotic course, respectively. * Indicates a gecko that did not receive antibiotic treatment due to logistical limitations. Note this diagram does not include geckos in the subcutaneous inoculation group as they did not develop clinical disease signs, and naturally infected geckos (H-group animals). 

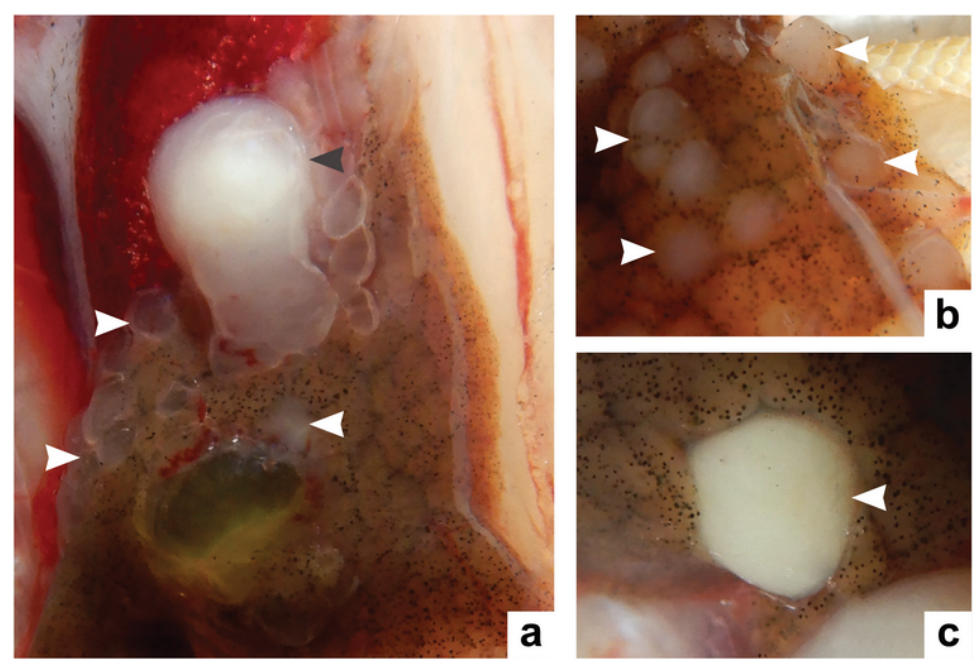

a
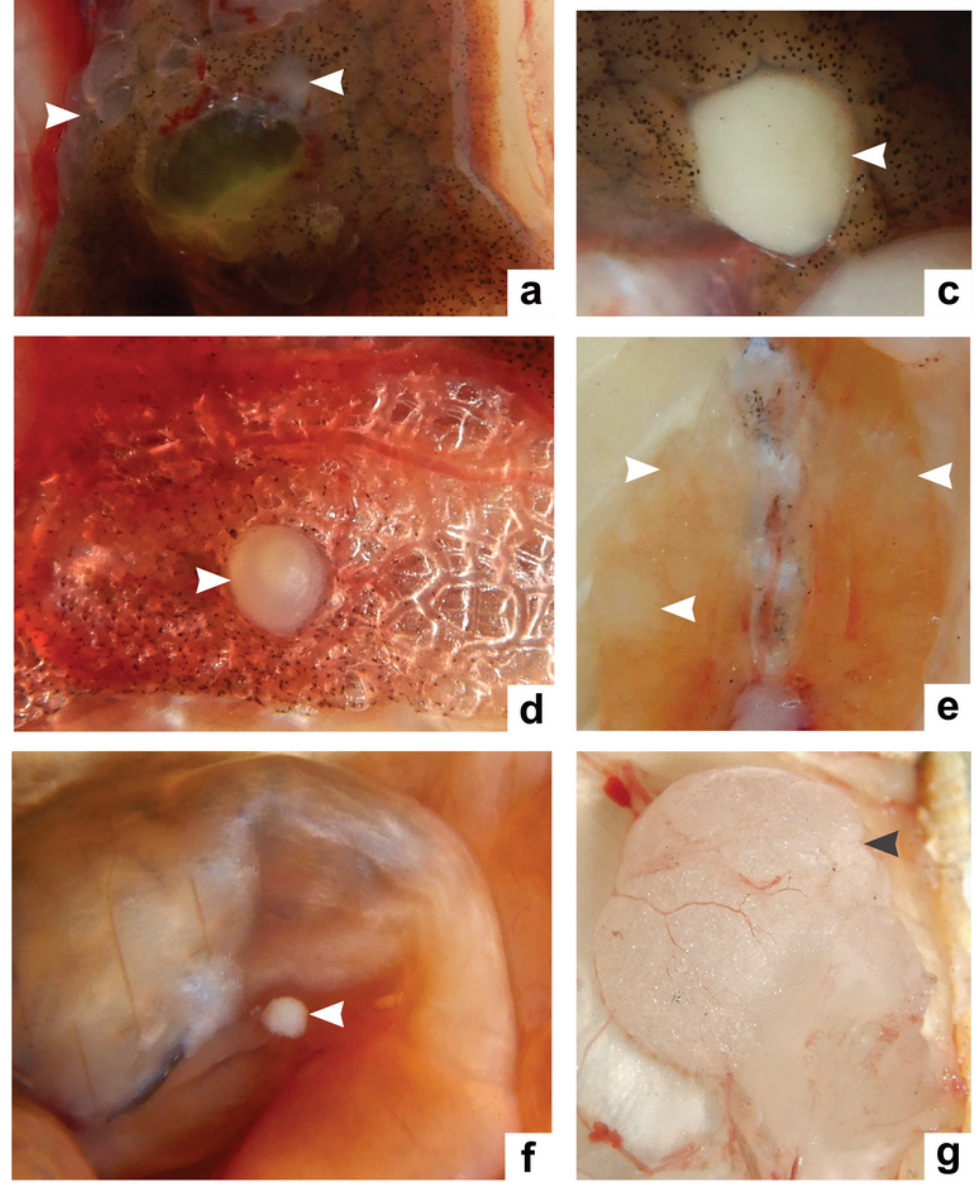

\section{Figure 3}

Macroscopic findings of Asian house geckos infected with E. lacertideformus. One large raised white focus adherent to the surface of the lung (grey arrowhead) and multiple smaller foci extending from the surface of the liver (white arrowheads) (gecko ID: CoC-4) (a), multifocal masses extensively replacing the hepatic parenchyma (white arrowheads) (gecko ID: SkL-2) (b), replacement of the gallbladder lumen with bacterial colonies (white arrowhead) (gecko ID: SuC-3) (c), a single raised white focus replacing parenchyma of the left lung and distending the pleura (white arrowhead) (gecko ID: Nal-9) (d), bilateral replacement of renal parenchyma by multiple raised white foci (white arrowheads) (gecko ID: SkL-2) (e), single raised white focus adhered to serosa of the lesser curvature of the stomach (white arrowhead) (gecko ID: SkL-2) (f), single large ventral mass cranial to pelvis (grey arrowhead) (gecko ID: CoC-3) (g). 


\section{Prior to Inoculation}

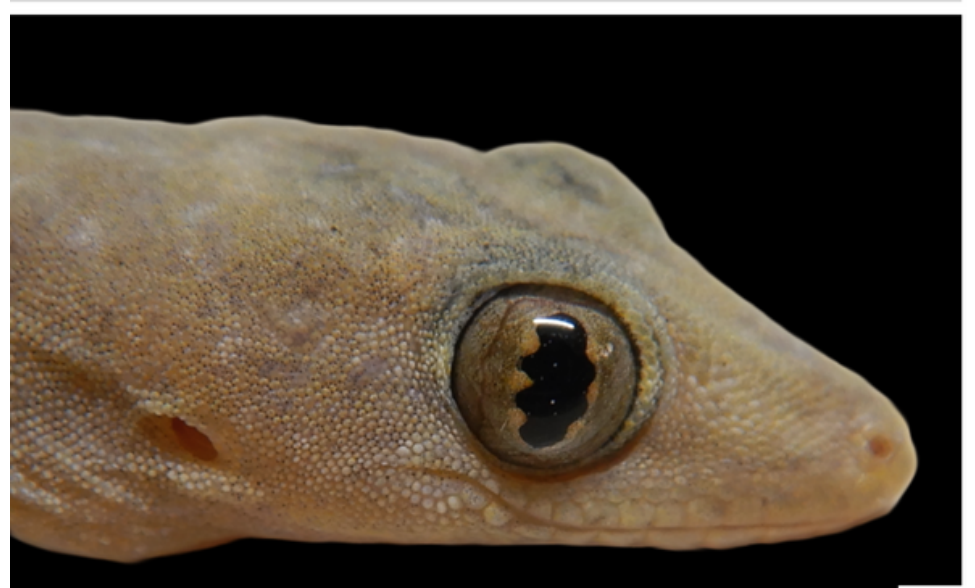

a

\section{Treatment Day 11}

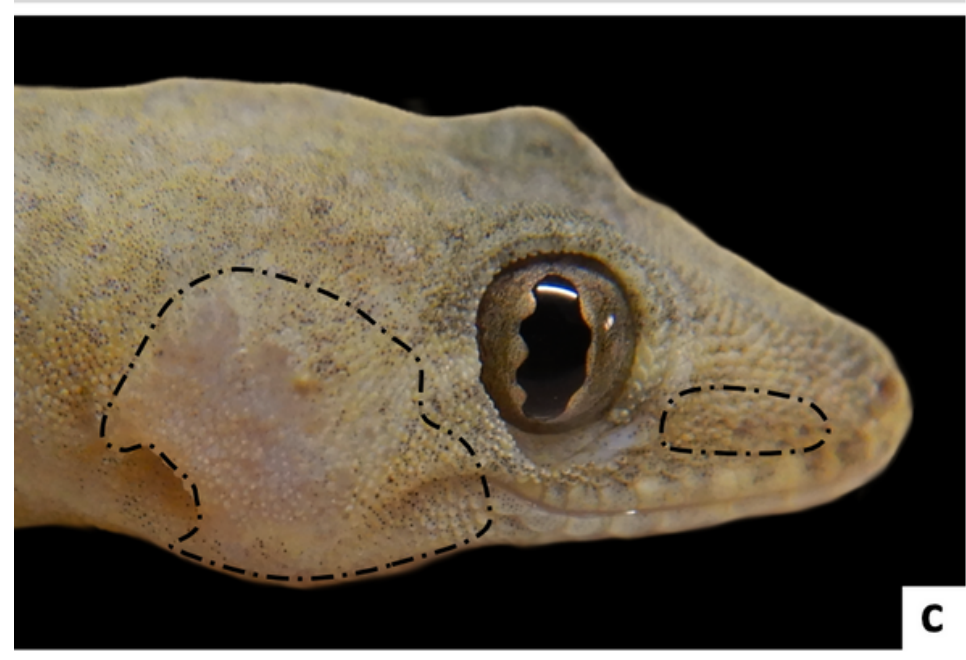

\section{Figure 4}

Treatment timeline of an Asian house gecko (gecko ID: SkL-4) experimentally infected with E. lacertideformus where lesions are delimited with a black line to illustrate the near complete regression of gross disease following treatment with enrofloxacin. Gecko prior to experimental inoculation via skin laceration at the right cheek (a). Representative lesions of E. lacertideformus observed at one (b), eleven (c), and 21 days (d) of oral antibiotic treatment with enrofloxacin. Near complete regression of gross lesions during antibiotic treatment is apparent, with markedly reduced subcutaneous swelling of the right cheek caudal to the eyes and cranial to the ear canal ostium (b-d). 


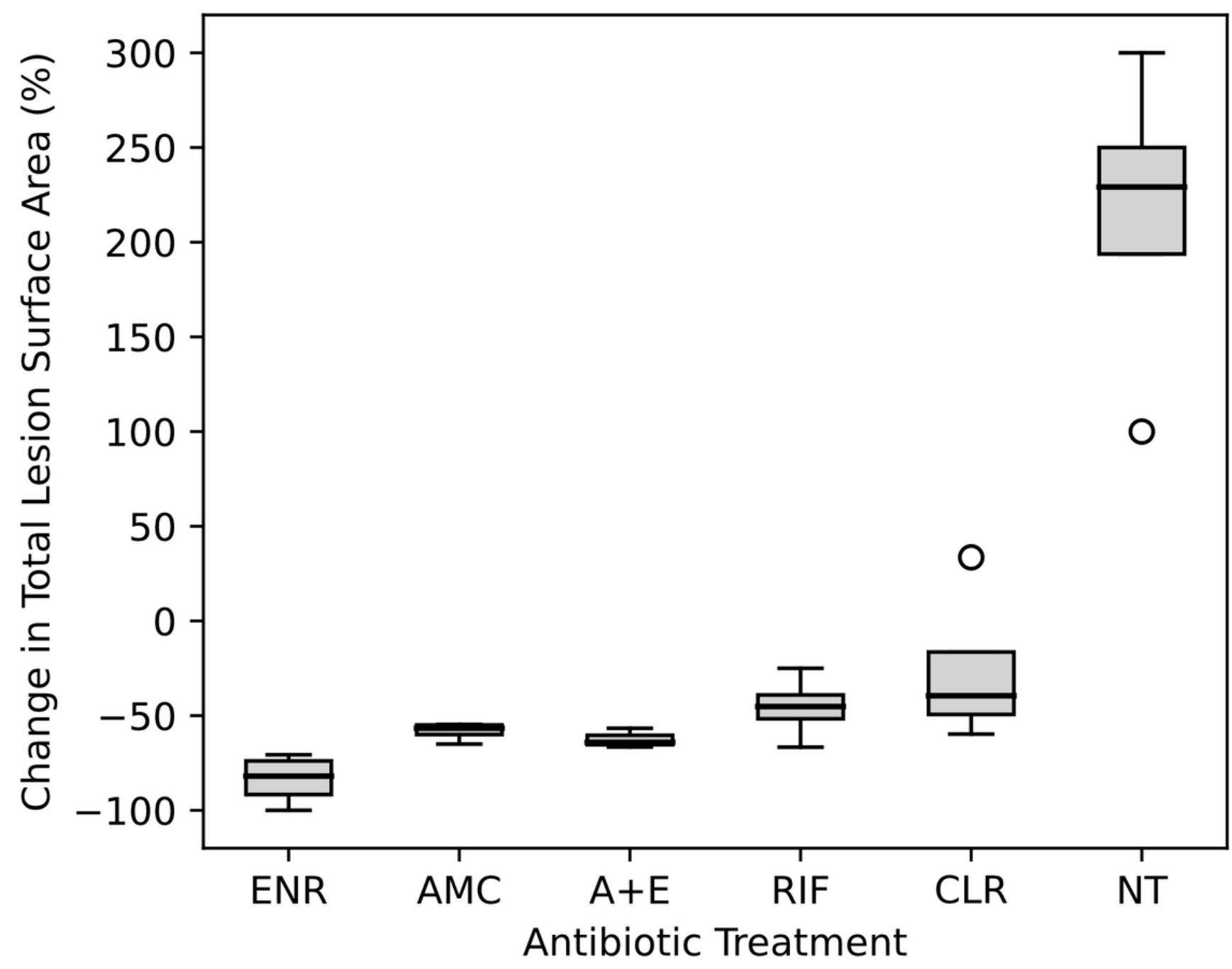

Figure 5

Boxplot of the average percentage change in total lesion surface area for each antibiotic treatment administered. The severity of each lesion was scored from 0 to 4 (the percentage replacement of normal tissue volume); where $0=$ no lesion present, $1=\mathrm{a}$ mild lesion ( $1-25 \%), 2=a$ moderate lesion $(25-50 \%), 3=$ a severe lesion (51 - 74\%), and $4=$ an extensive lesion (75 - 100\%). The bold line indicates the median, interquartile range (25th to 75 th percentile) is represented by the grey shading, the whiskers represent the minimum and maximum, and the outlier is shown by the circle. ENR, Enrofloxacin; AMC, Amoxicillin clavulanic acid; A+E, Amoxicillin clavulanic acid, and enrofloxacin; RIF, Rifampicin; CLR, Clarithromycin; NT, No treatment. 


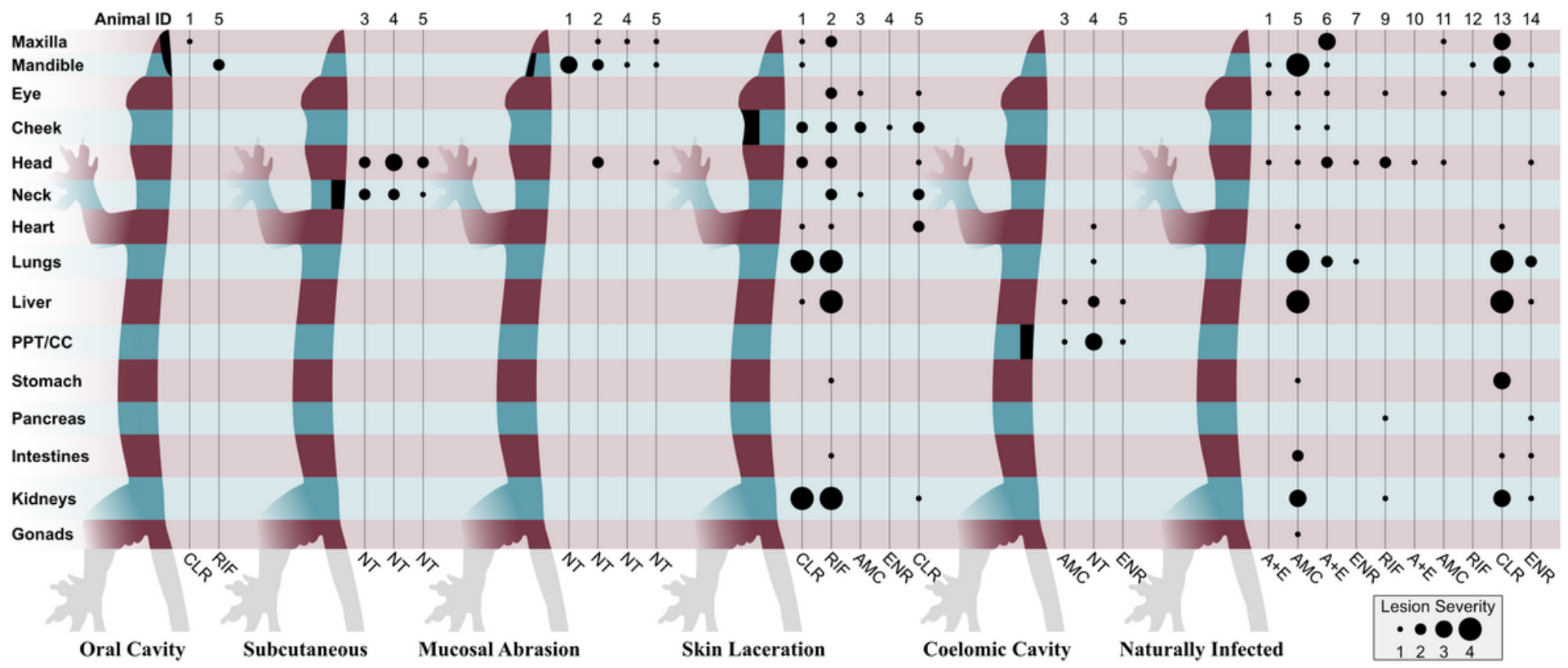

Figure 6

Histological distribution of E. lacertideformus in experimentally infected Asian house geckos per inoculation route and treatment group. The numerical values positioned at the top of the figure represent the ID of each infected gecko. The treatment administered to each gecko are abbreviated; AMC, Amoxicillin clavulanic acid; A+E, Amoxicillin clavulanic acid, and enrofloxacin; CLR, Clarithromycin; ENR, Enrofloxacin; NT, No treatment; RIF, Rifampicin. PPT/CC represents lesions associated with the pleural and pericardial tissues and/or the coelomic cavity. The black shading of the gecko represents the site of inoculation for that group. Identification of E. lacertideformus bacteria in organs/tissues are represented by the black circles. The severity of the histological lesions from 1 to 4 are denoted by the size of the circle. 


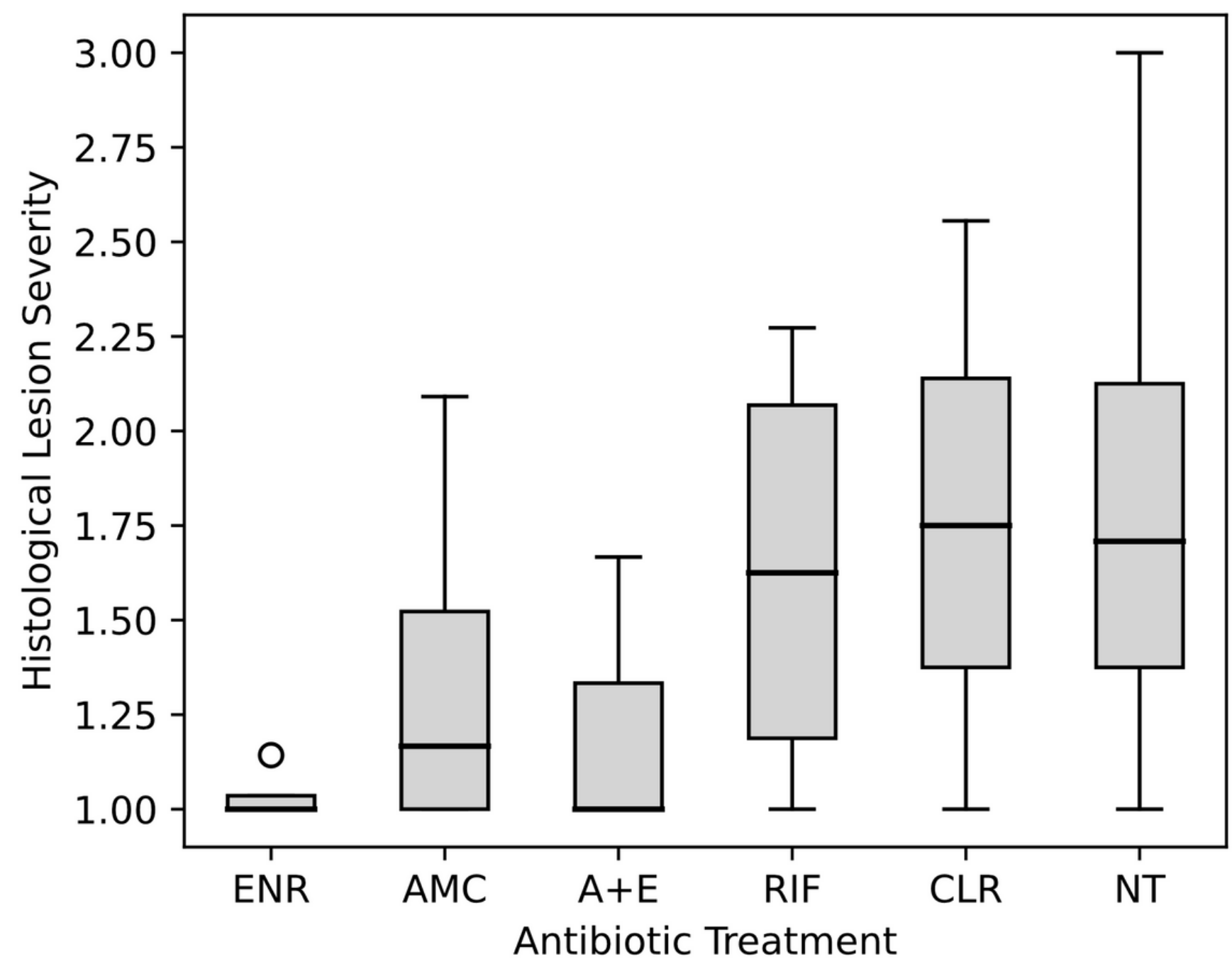

Figure 7

Boxplot of the average histological lesion severity for each antibiotic treatment administered. The bold line indicates the median, interquartile range (25th to 75th percentile) is represented by the grey shading, the whiskers represent the minimum and maximum, and the outlier is shown by the circle. ENR, Enrofloxacin; AMC, Amoxicillin clavulanic acid; A+E, Amoxicillin clavulanic acid, and enrofloxacin; RIF, Rifampicin; CLR, Clarithromycin; NT, No treatment.

\section{Supplementary Files}

This is a list of supplementary files associated with this preprint. Click to download.

- SupplementaryTablesTrialManuscript.docx 